\title{
Efecto de la adición de mezclas de ceniza volante y ceniza de fondo procedentes del carbón en la resistencia mecánica y porosidad de cementos Portland
}

\section{Effect of mixes made of coal bottom ash and fly ash on the mechanical strength and porosity of Portland cement}

\author{
C. $\operatorname{Argiz}^{(*, * *)}$, E. Menéndez(*), M. A. Sanjuán(***)
}

Recepción/Received: 10-VI-11

Aceptación/Accepted: 27-I-12

\section{RESUMEN}

La utilización de nuevas adiciones en el cemento es necesaria con el fin de obtener un material más sostenible y durable. En este sentido, las cenizas de fondo o cenicero de las centrales termoeléctricas de carbón se podrían reciclar siendo empleadas como un componente principal de los cementos Portland.

Se han estudiado las propiedades mecánicas de unos morteros normalizados elaborados con mezclas de cenizas volantes con cenizas de fondo fabricados con unos porcentajes similares a los correspondientes de los CEM II/A-V, CEM II/B-V y CEM IV/A (V).

En conclusión, la utilización de mezclas de cenizas de fondo o cenicero con cenizas volantes sustituyendo a éstas últimas entre el $0 \%$ y el $100 \%$, no influye significativamente en el comportamiento mecánico de los morteros estudiados en los que el contenido máximo de adición ha sido del 35\%, si bien afecta a determinados aspectos microestructurales, como la cantidad y distribución de poros capilares.

Palabras clave: cenizas de fondo; cenizas volantes; componentes del cemento; adiciones; resistencia a compresión.
SUMMARY

New additions to the cement are needed to achieve a more sustainable and durable construction material. Within this context, bottom ashes can be used as a main constituent of Portland cements when it is mixed in an optimized proportion with fly ashes.

The mechanical characteristics of standarized mortars made of mixes of pulverized coal combustion bottom and fly ashes are studied. The mortars were made of ordinary Portland cement (CEM I $42.5 \mathrm{~N}$ ) and mixes of bottom ashes with fly ashes in similar proportions to those of CEM II/A-V, CEM II/B-V and CEM IV/A (V).

Summing up, it can be said that the utilization of bottom ashes mixed with fly ashes in replacement levels from $0 \%$ to $100 \%$ do not affect significantively on the mechanical caracteristics of the mortars considered in the present study which had an addition maximum content of $35 \%$.

Keywords: bottom ash; fly ash; Portland cement constituents; additions; compressive strength.

(*) Instituto de Ciencias de la Construcción Eduardo Torroja (IETcc-CSIC) (Madrid, España).

(**) Universidad Politécnica de Madrid (Madrid, España).

(***) Instituto Español del Cemento y sus Aplicaciones (IECA) (Madrid, España). 


\section{INTRODUCCIÓN}

En Europa se ha puesto de manifiesto un significativo incremento en la utilización de adiciones en los cementos que se fabrican conforme con la norma de especificaciones EN 197-1:2011 (1) —en España actualmente la UNE-EN 197-1:2000 (2)-, lo cual se debe a razones medioambientales, por un lado, y económicas, por otro. Las adiciones más comúnmente utilizadas en España son las cenizas volantes silíceas (V), puzolanas naturales $(P)$, caliza ( $L$ y $L L)$ y escorias de horno alto (S) (3).

Las adiciones empleadas por la industria del cemento son muy diversas y presentan reactividades totalmente diferentes. El efecto de las adiciones normalizadas del cemento es bien conocido (4). Por ejemplo, la demanda de agua para obtener una docilidad del hormigón dada es menor en el caso de emplear cenizas volantes como consecuencia de su morfología esférica. En cuanto a la reacción puzolánica ésta se produce entre la sílice y la alúmina de las cenizas volantes y la portlandita, $\mathrm{Ca}(\mathrm{OH})_{2}$, procedente de la hidratación de los silicatos $\left(\mathrm{C}_{3} \mathrm{~S}\right.$ y $\left.\mathrm{C}_{2} \mathrm{~S}\right)$ del clínker del cemento Portland. Esta reacción es más lenta que la hidratación de los silicatos de calcio del clínker de cemento Portland por lo que necesita un curado más prolongado. Asimismo, las cenizas volantes juegan un papel muy importante rellenando parcialmente los poros de la pasta de cemento y las interfases árido-pasta y armadura-pasta, lo cual contribuye a una mayor durabilidad $y$, en algunos casos, a una mayor resistencia a largas edades siempre y cuando el hormigón se haya curado en condiciones de humedad $(4,5)$. Por su parte, los hormigones fabricados con cenizas volantes presentan condiciones durables adecuadas, por ejemplo, en aspectos tales como la resistencia a los ciclos de hielo-deshielo (6).

Las cenizas de fondo o cenicero se producen conjuntamente en la caldera de las centrales termoeléctricas de carbón por lo que su composición química en la mayoría de los casos es bastante similar. Hay pocos trabajos científicos publicados de estudios realizados con las cenizas de cenicero $(7,8)$ y, en su mayoría, tratan de su aplicación sin moler como áridos artificiales en sub-bases de carreteras $(9,10)$. El destino habitual de este residuo en los países occidentales ha sido el vertedero debido a la existencia de barreras técnicas, legales, regulatorias, contractuales, económicas - ya que hay que molerlas- y a una errónea percepción de riesgo en la seguridad y en la salud (11).

La mayor diferencia entre ambas cenizas se encuentra en su morfología y en el tamaño de las partículas, por este motivo, en este trabajo se pretende estudiar el

\section{INTRODUCTION}

In Europe, a clear increase in the use of additions in cements produced according to EN 197-1:2011 (1) Currently in Spain UNE-EN 197-1:2000 (2) - has been revealed. This is due not only to environmental reasons, but also to economical ones. The additions more frequently used in Spain are siliceous fly ash (V), natural pozzolans $(P)$, limestone ( $L$ or $L L)$ and ground granulated blast-furnace slag (S) (3).

The types of additions used in cement industry is wide and they present quite different reactivity. The effects of the standardized additions are well known (4). For instance, the water demand when fly ash ( $V$ ) is used is lower as a result of the spherical morphology. With regard to the pozzolanic reaction, the silica from the fly ash reacts together with the portlandite, $\mathrm{Ca}(\mathrm{OH})_{2}$, produced during the calcium silicates $\left(C_{3} S \& C_{2} S\right)$ hydration. Such calcium silicates are the normal constituents of Portland clinker. This reaction is slower than that of the calcium silicates $\left(C_{3} S \& C_{2} S\right.$ ) hydration mentioned above, therefore, a longer curing time is necessary. Fly ashes also play an important role filling partially the pores of the cement paste and the aggregate-paste and reinforcement-paste interfaces. This effect contributes to enhance the material durability and, in some cases, the material compressive strength at long ages, only when concrete has been cured in wet conditions $(4,5)$. On the other hand, concrete made of fly ashes exhibit an adequate durable condition. For instance, in concrete resistance to frost-thaw (6).

Fly ash and bottom ash are generated together in the boiler of coal fired power plants, and then, in most of the cases both ashes chemical composition is quite similar. There are only a few scientific papers published regarding studies performed on coal bottom ashes $(7,8)$. In most of them, coal bottom ashes are used as artificial aggregates in road bases $(9,10)$. The end of this waste in occidental countries in the past was landfilled due to several barriers (technical, regulatory, contractual, economical - grinding is costly - and so on) and to a wrong perception of risk on health and safety (11).

The main difference between both ashes is found in their morphology and grain size. Thus, the present paper tries to study the comparative performance of 
comportamiento comparativo de mezclas de cenizas volantes y cenizas de cenicero con relación a la resistencia mecánica de morteros normalizados fabricados con ellas y relacionarlo con la evolución de su microestructura. Asimismo, se han analizado algunos aspectos microestructurales mediante porosimetría de intrusión de mercurio (MIP) y absorción de agua.

\section{PARTE EXPERIMENTAL}

\subsection{Materiales}

Se empleó un cemento CEM I 42,5 N de la UNE-EN 197-1:2000 (2) como material de referencia y como materia prima para elaborar los diferentes cementos de adición de cenizas volantes y de cenicero. Los morteros de ensayo se fabricaron con arena normalizada alemana NORMSAND y agua destilada.

Se utilizaron las cenizas volantes y de cenicero de la central termoeléctrica de ENDESA-Carboneras (Almería) debido a las adecuadas propiedades químicas y físicas de la ceniza volante. Esta central termoeléctrica tiene una potencia de $1.158 .900 \mathrm{Kw}$ y consume carbón de Suráfrica $(90 \%)$ y Colombia (10\%). Las cenizas de cenicero o de fondo se molieron a la finura del cemento (residuo del 5-8\% en el tamiz de $45 \mu \mathrm{m})$.

\subsubsection{Composición química y características físicas}

La composición química de las cenizas volantes y de cenicero se presenta en la Tabla 1 . En esta misma Tabla 1 se recogen tanto las características físicas como químicas del CEM I 42,5 N. El análisis químico se realizó mediante espectrometría de fluorescencia de rayos $X$. La resistencia a compresión se determinó conforme la UNE-EN 196-1:2005; la pérdida por calcinación, residuo insoluble y contenido de cloruros conforme con la norma UNE-EN 196-2:2006; la determinación del inicio y final de fraguado y la estabilidad de volumen se ha realizado conforme con la norma UNE-EN 196-3:2005/ A1:2008 equivalente a la ISO 9597:2008 mientras que la densidad y superficie específica Blaine se determinó conforme con la norma UNE-EN 196-6:2010.

La Figura 1 muestra el estudio granulométrico del cemento y de las cenizas volantes y de cenicero que se realizó por granulometría láser (Malvern Instruments, Ltd. Mastersize 2000 Version 5.40 - Fraunhofer Method). Estas últimas fueron molidas en un molino de bolas de laboratorio de unos 48 litros de capacidad hasta conseguir un residuo del $5-8 \%$ en el tamiz de $45 \mu \mathrm{m}$ con el fin de obtener una finura similar a la del cemento. fly ashes and bottom ashes mixes with regard to the mechanical strength of standard mortars made of them and link it with the microstructural development. Likewise, some microstructural aspects were analysed by means of mercury intrusion porosimetry (MIP) and water absorption.

\section{EXPERIMENTAL}

\subsection{Materials}

A CEM I $42.5 \mathrm{~N}$ cement according to the European Standard EN 197-1:2011 (1) was used as reference material and also as raw material to prepare in the laboratory blended cements made of fly ash and bottom ash. Testing mortars were prepared with German standard sand (NORMSAND) and distilled water.

The fly ash and bottom ash were provided by ENDESACarboneras (Almería - Andalucía). They were chosen due to the chemical and physical properties of the fly ash. The power station has a capacity of 1.158 .900 $\mathrm{KW}$ and it uses hard coal from South Africa (90\%) and Colombia (10\%). Bottom ash was ground to fineness similar to that of the Portland cement (5-8\% residue on $45 \mu m$ sieve).

\subsubsection{Chemical composition and physical characteristics}

The chemical composition of the fly ash and bottom ash are shown in Table 1. Physical characteristics of cements CEM I $42.5 \mathrm{~N}$ (EN 197-1:2011) are also shown in Table 1. The chemical analysis was performed by XRF. The compressive strength was determined according to the Spanish standard UNE-EN 196-1:2005 (Equivalent to the European one EN 196-1). The loss on ignition, insoluble residue and chloride content were determined according to UNE-EN 196-2:2006. The initial and final setting times and the expansion were determined according to UNEEN 196-3:2005/A1:2008, equivalent to ISO 9597:2008; while density and specific surface (Blaine) were determined according to UNE-EN 196-6:2010.

Figure 1 shows the granulometric study of cements, fly ash and bottom ash, which was performed by laser granulometry (Malvern Instruments, Ltd. Mastersize 2000 Version 5.40 - Fraunhofer Method). Bottom ash was ground in a laboratory ball mill of 48 litres of capacity in order to reach a 5-8\% residue on $45 \mu \mathrm{m}$ sieve (similar to cement fineness). 
Tabla 1 / Table 1

Composición química de las cenizas volantes (V), de las cenizas de cenicero o fondo (C) y del cemento sin adición CEM I 42,5 N de la UNE-EN 197-1:2011 utilizado como referencia.

Chemical composition of fly ash (V), bottom ash (C) and cement CEM I 42.5N (EN 197-1:2011) without any.

\begin{tabular}{|c|c|c|c|c|c|}
\hline $\begin{array}{l}\text { Composición química / } \\
\text { Chemical composition (\%) }\end{array}$ & $\begin{array}{c}\text { Cemento I } \\
\text { Cement }\end{array}$ & $\begin{array}{l}\text { Cenizas } \\
\text { volantes I } \\
\text { Fly ash (V) }\end{array}$ & $\begin{array}{c}\text { Cenizas de cenicero } \\
\text { o de fondo / } \\
\text { Bottom ash (C) }\end{array}$ & \multicolumn{2}{|c|}{$\begin{array}{l}\text { Propiedades físicas del cemento I } \\
\text { Cement physical properties }\end{array}$} \\
\hline $\mathrm{SiO}_{2}$ & 20.9 & 50.5 & 52.2 & Densidad/Density $\left(\mathrm{kg} / \mathrm{m}^{3}\right)$ & 3.10 \\
\hline $\mathrm{Al}_{2} \mathrm{O}_{3}$ & 4.3 & 28.9 & 27.5 & $\begin{array}{l}\text { Inicio del tiempo de fraguado / } \\
\text { Initial setting time (min) }\end{array}$ & 205 \\
\hline $\mathrm{Fe}_{2} \mathrm{O}_{3}$ & 3.5 & 4.7 & 6.0 & $\begin{array}{l}\text { Final del tiempo de fraguado / } \\
\text { Final setting time (min) }\end{array}$ & 325 \\
\hline $\mathrm{CaO}$ & 62.7 & 5.0 & 5.9 & Expansión/Expansion (mm) & 0.70 \\
\hline $\mathrm{MgO}$ & 1.9 & 1.8 & 1.7 & $\begin{array}{c}\text { Superficie específica Blaine / } \\
\text { Blaine specific surface area }\left(\mathrm{m}^{2} / \mathrm{kg}\right)\end{array}$ & 4050 \\
\hline $\mathrm{SO}_{3}$ & 3.4 & 0.21 & 0.13 & $\begin{array}{l}\text { Resistencia a compresión/ } \\
\text { Compressive strength }\end{array}$ & $(\mathrm{MPa})$ \\
\hline $\mathrm{K}_{2} \mathrm{O}$ & 0.9 & 0.80 & 0.57 & 1 día / day & 13.30 \\
\hline $\mathrm{Ti}_{2} \mathrm{O}_{5}$ & 0.25 & 1.56 & 1.53 & 3 días / days & 19.45 \\
\hline $\mathrm{P}_{2} \mathrm{O}_{5}$ & 0.10 & 0.76 & 0.74 & 7 días / days & 37.95 \\
\hline $\begin{array}{l}\text { Pérdida por calcinación / } \\
\text { Loss on ignition }\end{array}$ & 3.69 & 3.6 & 1.8 & 14 días / days & 45.25 \\
\hline $\begin{array}{l}\left.\text { Residuo insoluble (Método del } \mathrm{Na}_{2} \mathrm{CO}_{3}\right) / \\
\text { Insoluble residue }\left(\mathrm{Na}_{2} \mathrm{CO}_{3} \text { Method) }\right.\end{array}$ & 1.04 & 71.3 & 75.7 & 28 días / days & 50.98 \\
\hline $\mathrm{Cl}^{-}$ & 0.023 & 0.00 & 0.001 & 90 días / days & 55.55 \\
\hline
\end{tabular}

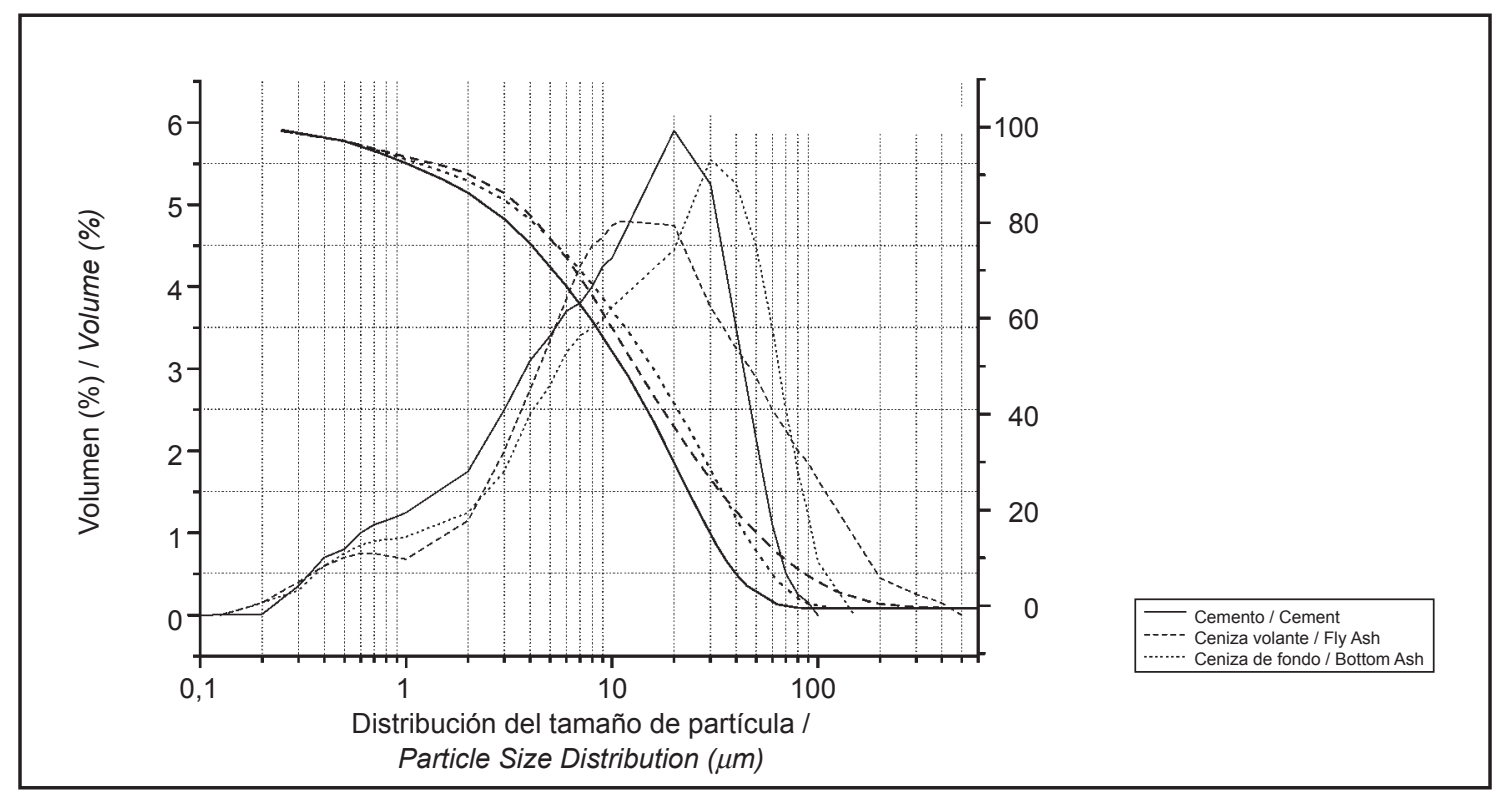

Figura 1. Estudio granulométrico del cemento y de las cenizas volantes y de cenicero. Figure 1. Granulometric study of cement, fly ash and bottom ash.

\subsection{Mezcla de cenizas}

Se han utilizado cinco mezclas de cenizas volantes (V) y de cenizas de cenicero $(C)$ codificadas con las letras $\beta, \gamma, \delta, \lambda$ y $\Omega$ (Tabla 2). En estas mezclas se reemplazó parte del cemento CEM I 42,5 N, codificado con la letra $\alpha$, para formar las diferentes mezclas conglomerantes

\subsection{Ash mixes}

Five fly and bottom ashes mixes were prepared and coded as $\beta, \gamma, \delta, \lambda$ and $\Omega$ (Table 2). Cement was coded as $\alpha$ (CEM I $42.5 \mathrm{~N})$ and it was partially replaced by the five ashes mixes mentioned above, in order to make binders with similar compositions to the CEM II/A-V*, 
similares a las de los cementos CEM II/A-V*, CEM II/BV* y CEM IV/A $\left(\mathrm{V}^{*}\right)$, las cuales se compararon con el CEM I 42,5 N sin adiciones. El símbolo ( $\mathrm{V}^{*}$ ) corresponde a la mezcla de cenizas volantes $(V)$ con cenizas de cenicero (C).

La Tabla 2 muestra la dosificación y la denominación de las mezclas de cenizas volantes (V) y de cenizas de cenicero (C) estudiadas. Se han utilizado mezclas de cenizas volantes $(\beta)$ y cenizas de cenicero $(\Omega)$, en proporciones $90 \%: 10 \%(\gamma), 80 \%: 20 \%(\delta)$ y $50 \%: 50 \%$ $(\lambda)$. La elección de las dosificaciones estudiadas se justifica por el hecho de que, del total de cenizas producidas en una central termoeléctrica de carbón —cenizas volantes (V) y cenizas de cenicero (C)-; en Europa, según ECOBA (12) en 2005, el 87,5\% son cenizas volantes (V) y el $12,5 \%$ son cenizas de cenicero (C), mientras que en USA según el ACAA en 2007 (13), estos mismos valores se sitúan en el $80 \%$ (V) y el $20 \%$ (C), respectivamente.
CEM II/B-V* and CEM IV/A $\left(V^{*}\right)$. Their properties were compared to those of CEM I $42.5 \mathrm{~N}$ (without additions). The symbol $\left(V^{*}\right)$ means a mixture of fly ash $(V)$ and bottom ash (C).

Table 2 shows the composition and denomination of the fly ash ( $V$ ) and bottom ash (C) mixes. Fly ash ( $\beta$ ) and bottom ash $(\Omega)$ were mixed in proportions $90 \%: 10 \%$ $(\gamma), 80 \%: 20 \%(\delta)$ and $50 \%: 50 \%(\lambda)$. The election of the proportions studied is justified as a coal fired power plant in Europe in 2005 produces an average of $87.5 \%$ of fly ashes (V) and $12.5 \%$ of bottom ash (C) according to ECOBA (12), whereas in the USA the same ratio is $80 \%(\mathrm{~V})$ and $20 \%(\mathrm{C})$, respectively (ACAA in 2007 (13)).

Tabla 2 / Table 2

Proporciones y denominación de las mezclas de las cenizas volantes (V) con las cenizas de cenicero o de fondo (C) / Proportions and denomination of ash mixes containing fly ash (V) and/or bottom ash (C).

\begin{tabular}{|c|c|c|c|c|c|c|}
\hline \multirow{2}{*}{ \% Material / Material } & \multicolumn{5}{|c|}{ Denominación de las mezclas de cenizas / Ash mixes code } \\
\cline { 2 - 7 } & $\boldsymbol{\alpha}$ & $\boldsymbol{\beta}$ & $\mathrm{Y}$ & $\boldsymbol{\delta}$ & $\boldsymbol{\lambda}$ & $\boldsymbol{\Omega}$ \\
\hline Ceniza volante / Fly ash (V) & $0 \%$ & $100 \%$ & $90 \%$ & $80 \%$ & $50 \%$ & $0 \%$ \\
\hline Ceniza de cenicero / Bottom ash (C) & $0 \%$ & $0 \%$ & $10 \%$ & $20 \%$ & $50 \%$ & $100 \%$ \\
\hline
\end{tabular}

\subsection{Mezcla y elaboración de morteros}

Las mezclas de los componentes del cemento (cenizas volantes $(\mathrm{V})$, cenizas de cenicero (C) y el cemento CEM I 42,5 $\mathrm{N}$ sin adiciones) se han realizado de forma tal que se han obtenido los siguientes tipos de cementos:

$$
\begin{gathered}
\text { CEM I: } 0 \%(\mathrm{~V}+\mathrm{C}) \\
\text { CEM II/A-V*: } 10 \%(\mathrm{~V}+\mathrm{C}) \\
\text { CEM II/B-V*: } 25 \%(\mathrm{~V}+\mathrm{C}) \\
\text { CEM IV/A }\left(\mathrm{V}^{*}\right): 35 \%(\mathrm{~V}+\mathrm{C})
\end{gathered}
$$

La Tabla 3 resume los cuatro tipos de cemento considerados junto con las cinco mezclas de cenizas volantes (V) y cenizas de cenicero (C) además del patrón sin adición $(\alpha)$. Con estas mezclas de cenizas y cemento CEM I 42,5 $\mathrm{N}$ se han fabricado los diferentes morteros de ensayo empleando la arena normalizada alemana NORMSAND y agua destilada.

Los morteros de ensayo se elaboraron con los componentes indicados en la Tabla 3 conforme con la norma UNE-EN 196-1:2005 (14). Por tanto, la dosificación de

\subsection{Mortars mix and elaboration}

After mixing ashes (bottom ash and fly ash) with cement CEM I $42.5 \mathrm{~N}$, the following cement types were obtained:

\author{
CEM I : $0 \%(V+C)$ \\ CEM II/A-V* : $10 \%(V+C)$ \\ CEM II/B- $V^{*}: 25 \%(V+C)$ \\ CEM IV/A $\left(V^{*}\right): 35 \%(V+C)$
}

Table 3 summarises the four cement types elaborated in this research and the five mixes of fly ash $(V)$ and bottom ash (C), and also the reference cement without any addition $(\alpha)$. With these ash mixes and CEM I $42.5 \mathrm{~N}$ several mortars were prepared using standardized sand (NORMSAND-Germany) and distilled water.

The testing mortars were made with the components shown in Table 3 according to the European Standard EN 196-1:2005 (14). Therefore, the mix dosage has been: 
Tabla 3 / Table 3

Proporciones y denominación de las 16 mezclas de las cenizas volantes (V), cenizas de cenicero (C) y cemento CEM I 42,5 N sin adiciones.

Mix design and denomination of 16 mixes made of fly ash (V), bottom ash (C) and cement CEM I 42.5N without any addition.

\begin{tabular}{|c|c|c|c|c|c|c|c|c|}
\hline \multirow{2}{*}{$\begin{array}{l}\text { Tipos de cementos I } \\
\text { Cement types }\end{array}$} & \multirow{2}{*}{\multicolumn{2}{|c|}{$\begin{array}{c}\% \text { Mezcla de cenizas } / \\
\text { Ash mixes, } \%\end{array}$}} & \multicolumn{6}{|c|}{ Denominación de la mezcla de cenizas / Ash mixes code } \\
\hline & & & $\alpha$ & $\beta$ & y & $\delta$ & $\lambda$ & $\Omega$ \\
\hline \multirow{3}{*}{ CEM I } & Ceniza volante / Fly ash & \multirow[b]{2}{*}{$0 \%$} & \multirow[b]{2}{*}{$0 \%$} & & & & & \\
\hline & Ceniza fondo / Bottom ash & & & & & & & \\
\hline & Cemento / Cement & $100 \%$ & $100 \%$ & & & & & \\
\hline \multirow{3}{*}{ CEM II/A-V* } & Ceniza volante / Fly ash & \multirow[b]{2}{*}{$10 \%$} & & $10 \%$ & $9 \%$ & $8 \%$ & $5 \%$ & $0 \%$ \\
\hline & Ceniza fondo / Bottom ash & & & $0 \%$ & $1 \%$ & $2 \%$ & $5 \%$ & $10 \%$ \\
\hline & Cemento / Cement & $90 \%$ & & $90 \%$ & $90 \%$ & $90 \%$ & $90 \%$ & $90 \%$ \\
\hline \multirow{3}{*}{ CEM II/B-V* } & Ceniza volante / Fly ash & \multirow[b]{2}{*}{$25 \%$} & & $25 \%$ & $22.5 \%$ & $20 \%$ & $12.5 \%$ & $0 \%$ \\
\hline & Ceniza fondo / Bottom ash & & & $0 \%$ & $2.5 \%$ & $5 \%$ & $12.5 \%$ & $25 \%$ \\
\hline & Cemento / Cement & $75 \%$ & & $75 \%$ & $75 \%$ & $75 \%$ & $75 \%$ & $75 \%$ \\
\hline \multirow{3}{*}{ CEM IVIA $\left(\mathrm{V}^{*}\right)$} & Ceniza volante / Fly ash & \multirow[b]{2}{*}{$35 \%$} & & $35 \%$ & $31.5 \%$ & $28 \%$ & $17.5 \%$ & $0 \%$ \\
\hline & Ceniza fondo / Bottom ash & & & $0 \%$ & $3.5 \%$ & $7 \%$ & $17.5 \%$ & $35 \%$ \\
\hline & Cemento / Cement & $65 \%$ & & $65 \%$ & $65 \%$ & $65 \%$ & $65 \%$ & $65 \%$ \\
\hline
\end{tabular}

estos morteros ha sido: relación agua/cemento $=0,50$ y relación cemento/arena $=1 / 3$. La conservación ha sido en cámara de curado a una H.R. $>90 \%$ y $20{ }^{\circ} \mathrm{C}$ de temperatura durante 24 horas y, posteriormente, curado bajo agua hasta su ensayo.

\subsection{Métodos de ensayo}

Los ensayos de resistencia a flexotracción y a compresión a 1, 3, 7, 14, 28 y 90 días de los morteros elaborados con el cemento patrón y las mezclas de las cenizas volantes con las de cenicero (de la $\beta$ a la $\Omega$ ) se ha realizado conforme con la norma UNE-EN 196-1:2005 (14) empleando una prensa IBERTEST modelo Autotest 200-10-5wc.

El estudio microestructural se ha realizado mediante porosimetría de intrusión de mercurio (MIP) con un equipo Micromeritics AutoPore IV 9500 V1.05 Serial 293 desde 0,50 hasta 33.000 psia. Asimismo, la porosidad abierta se ha determinado mediante absorción de agua conforme con la UNE-EN 1936:2007 (15). La granulometría de las cenizas y del cemento se determinó por granulometría láser con un equipo Malvern Instruments, Ltd. Mastersize 2000 Version 5.40 Fraunhofer Method.

\section{RESULTADOS}

\subsection{Resistencia a flexión}

La Figura 2 presenta los resultados de resistencia a flexión a 1, 3, 7, 14, 28 y 90 días de los morteros fabricados con las mezclas de cenizas volantes con las de cenicero (de la $\beta$ a la $\Omega$ ). Se muestran, de izquierda a derecha, los tres cementos de adición simulados CEM water/cement $=0.50$, and cement/sand $=1 / 3$. After mixing, the specimens were kept in a climate chamber at $>90 \%$ R.H. and $20{ }^{\circ} \mathrm{C}$ for 24 hours. Later on, they were kept under water until testing.

\subsection{Testing Procedure}

Compressive and flexure strength testing were performed on mortars at 1, 3, 7, 14, 28 and 90 days according to UNE-EN 196-1:2005 (14) by using an IBERTESTAUTOTEST 200-10-5 wc Model.

Microstructural analyses were developed by means of mercury intrusion porosimeter (MIP) from 0.50 to 3300 psia using a Micromeritics Autopore IV 9500 v1.05 serial 293. Open porosity was also determined by water absorption according to UNE-EN 1936:2007 (15). Ashes and cement granulometry was determined with a Mastesize 2000 V.5.40 laser granulometer (Malvern Instruments, Ltd.)

\section{RESULTS}

\subsection{Flexure strength}

Figure 2 shows the flexure strength results at 1, 3, 7, 14,28 and 90 days of mortars made of fly ash and bottom ash mixes (from $\beta$ to $\Omega$ ). From left to right, the three prepared in laboratory cements CEM II/A$V^{*}, C E M I I / B-V^{*}$ and CEM IV/A $\left(V^{*}\right)$ are shown in 


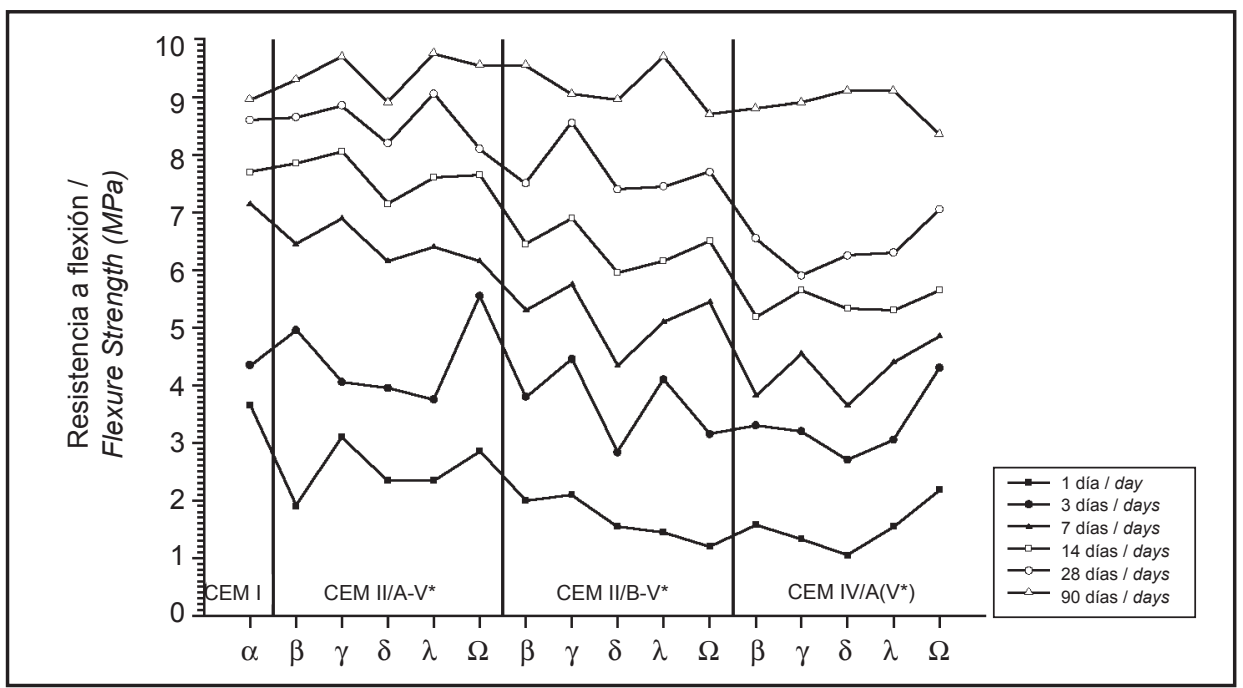

Figura 2. Resistencia a flexotracción a 1, 3, 7, 14, 28 y 90 días de los morteros con las mezclas de cenizas volantes con las de cenicero $(\beta \rightarrow \Omega)$. Se muestran, de izquierda a derecha, los tres cementos de adición simulados CEM II/A-V*, CEM II/B-V* y CEM IV/A ( $\left.V^{*}\right)$ en comparación con el cemento sin adiciones CEM I 42,5 N $(\alpha)$.

Figure 2. Flexure strength at 1, 3, 7, 14, 28 and 90 days of tested mortars made of fly ash and bottom ash mixes $(\beta \rightarrow \Omega)$ From left to right, laboratory-made blended cements CEM II/A- $V^{*}$, CEM II/B- $V^{*}$ and CEM IV/A( $\left.V^{*}\right)$ in comparison to CEM I $42.5 \mathrm{~N}(\alpha)$ without any addition are shown.

II/A-V*, CEM II/B-V* y CEM IV/A (V*) en comparación con el cemento sin adición CEM I 42,5 N ( $\alpha)$.

\subsection{Resistencia a compresión}

La Figura 3 presenta los resultados de resistencia a compresión a 1, 3, 7, 14, 28 y 90 días de los morteros elaborados con las mezclas de cenizas volantes con las de cenicero (de la $\beta$ a la $\Omega$ ). Se muestran, de izquierda a derecha, los tres cementos de adición simulados CEM II/A-V*, CEM II/B-V* y CEM IV/A ( $V *)$ en comparación con el cemento sin adición CEM I 42,5 N $(\alpha)$.

\subsection{Porosidad de los morteros}

La Figura 4 presenta los resultados de porosidad abierta determinada mediante la UNE-EN 1936:2007 (15) a las edades de 28 y 90 días en los morteros de adición fabricados con los conglomerantes que simulan los cementos CEM II/A-V*, CEM II/B-V* y CEM IV/A $\left(V^{*}\right)$ elaborados sólo con cenizas volantes $(\beta)$ o de cenicero $(\Omega)$ y con la mezcla $1: 1(\lambda)$ de ambas en comparación con los morteros fabricados con el cemento sin adición CEM I 42,5 N $(\alpha)$. Por otro lado, las Figuras 5 y 6 presentan los resultados microestructurales obtenidos mediante porosimetría de intrusión de mercurio (Mercury intrusión porosimetry -MIP-) a las edades de 28 y 90 días, respectivamente, en los mismos morteros ya citados. Finalmente, la Figura 7 presenta la porosidad total a las edades de 28 y 90 días, determinada mediante porosimetría de intrusión de mercurio. comparison to a cement without any addition CEM I $42.5 N(\alpha)$.

\subsection{Compressive strength}

Figure 3 shows the compressive strength results at 1, 3, $7,14,28$ and 90 days of the mortars made of fly ash and bottom ash mixes (from $\beta$ to $\Omega$ ). From left to right, the three elaborated cements CEM II/A-V*, CEM II/B-V* and CEM IV/A $\left(V^{*}\right)$ are shown in comparison to CEM I $42.5 \mathrm{~N}$ $(\alpha)$ without additions.

\subsection{Mortar Porosity}

Figure 4 shows the open porosity results determined by UNE-EN 1936:2007 (15) at 28 and 90 days, in mortars with CEM II/A-V*, CEM II/B-V* and CEM IV/A $\left(V^{*}\right)$ which were made of fly ash $(\beta)$, bottom ash $(\Omega)$ or a mixture $1: 1$ of both of them $(\lambda)$ in comparison to mortars made without any addition CEM I $42.5 \mathrm{~N}$ $(\alpha)$. On the other hand, Figures 5 and 6 show the microstructural results obtained by mercury intrusion porosimetry (MIP) at 28 and 90 days, respectively, in the same mortars mentioned above. Finally, Figure 7 shows the total porosity at 28 and 90 days determined by mercury intrusion porosimetry (MIP). 


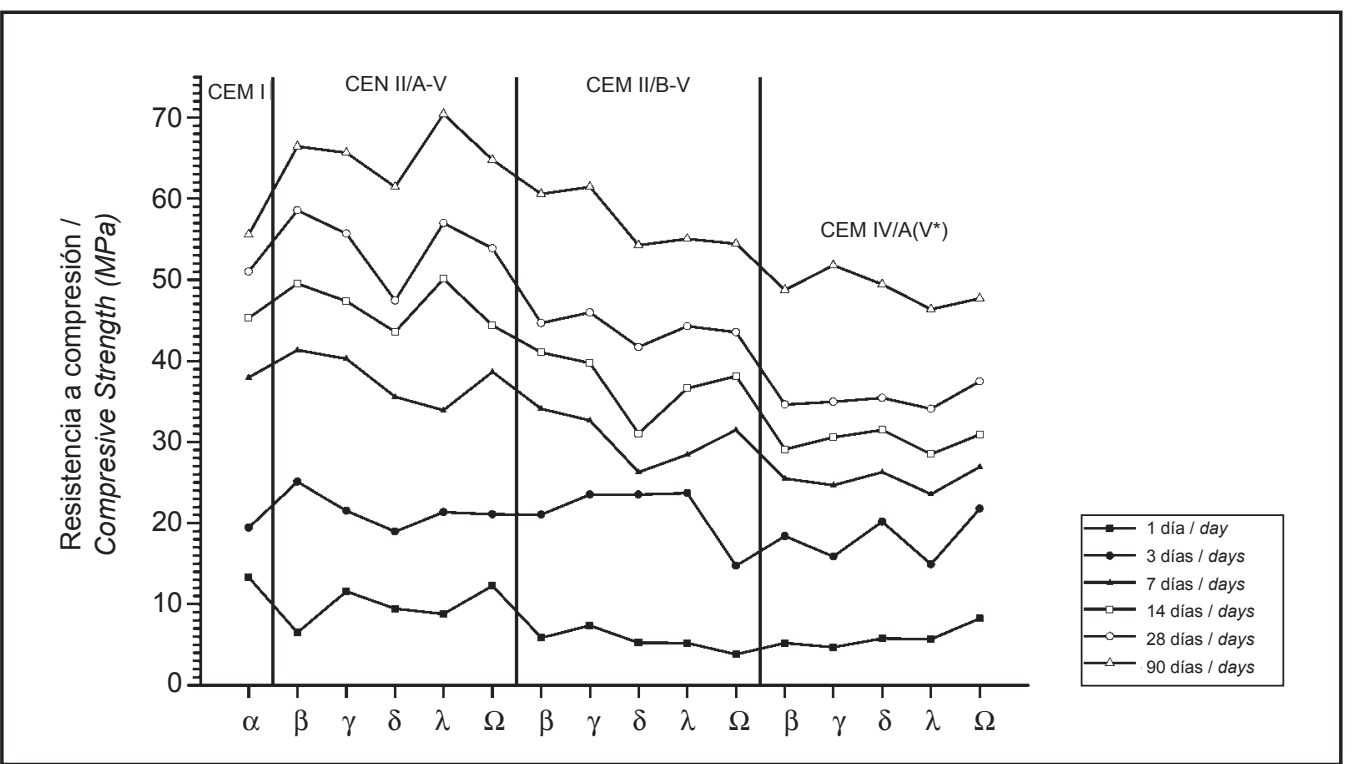

Figura 3. Resistencia a compresión a 1, 3, 7, 14, 28 y 90 días de los morteros con las mezclas de cenizas volantes con las de cenicero $(\beta \rightarrow \Omega)$. Se muestran, de izquierda a derecha, los tres cementos de adición simulados CEM II/A-V*, CEM II/B-V* y CEM IV/A ( $\left.V^{*}\right)$ en comparación con el cemento sin adiciones CEM I 42,5 N $(\alpha)$.

Figure 3. Compressive strength at $1,3,7,14,28$ and 90 days of tested mortars made of fly ash and bottom ash mixes $(\beta \rightarrow \Omega)$ From left to right, laboratory-made blended cements CEM II/A-V*, CEM II/B- $V^{*}$ and CEM IV/A(V*) in comparison to CEM I $42.5 \mathrm{~N}(\alpha)$ without any addition are shown.

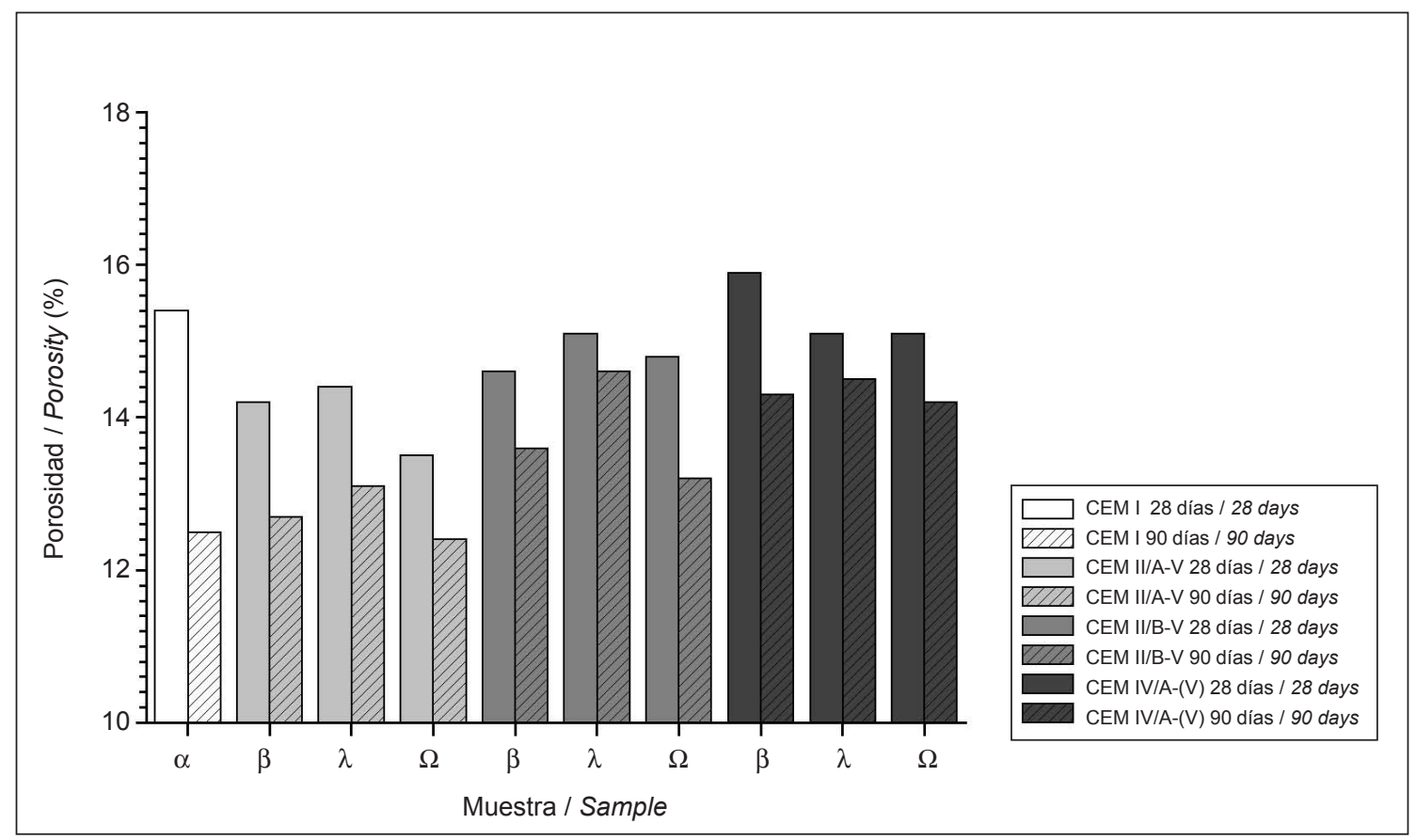

Figura 4. Porosidad abierta a 28 y 90 días de los morteros de adición simulando cementos CEM II/A-V*, CEM II/B-V* y CEM IV/A (V*) con cenizas volantes silíceas $(\mathrm{V}), \beta$, y cenizas de fondo (C), $\Omega$, y morteros con una mezcla 1:1 de ambas cenizas, l, en comparación con el cemento sin adiciones CEM I 42,5 N $(\alpha)$, determinada mediante absorción de agua (UNE-EN 1936:2007).

Figure 4. Open porosity at 28 and 90 days of blended mortars made of cements prepared in laboratory CEM II/A-V*, CEM II/B-V* and CEM IV/A $\left(V^{*}\right)$, which had fly ash $(V), \beta$, bottom ash $(C), \Omega$, and a 1:1 (fly ash: bottom ash) mix, in comparison to cement CEM I $42.5 \mathrm{~N}(\alpha)$ without any addition determined by water absorption (UNE-EN 1936:2007). 

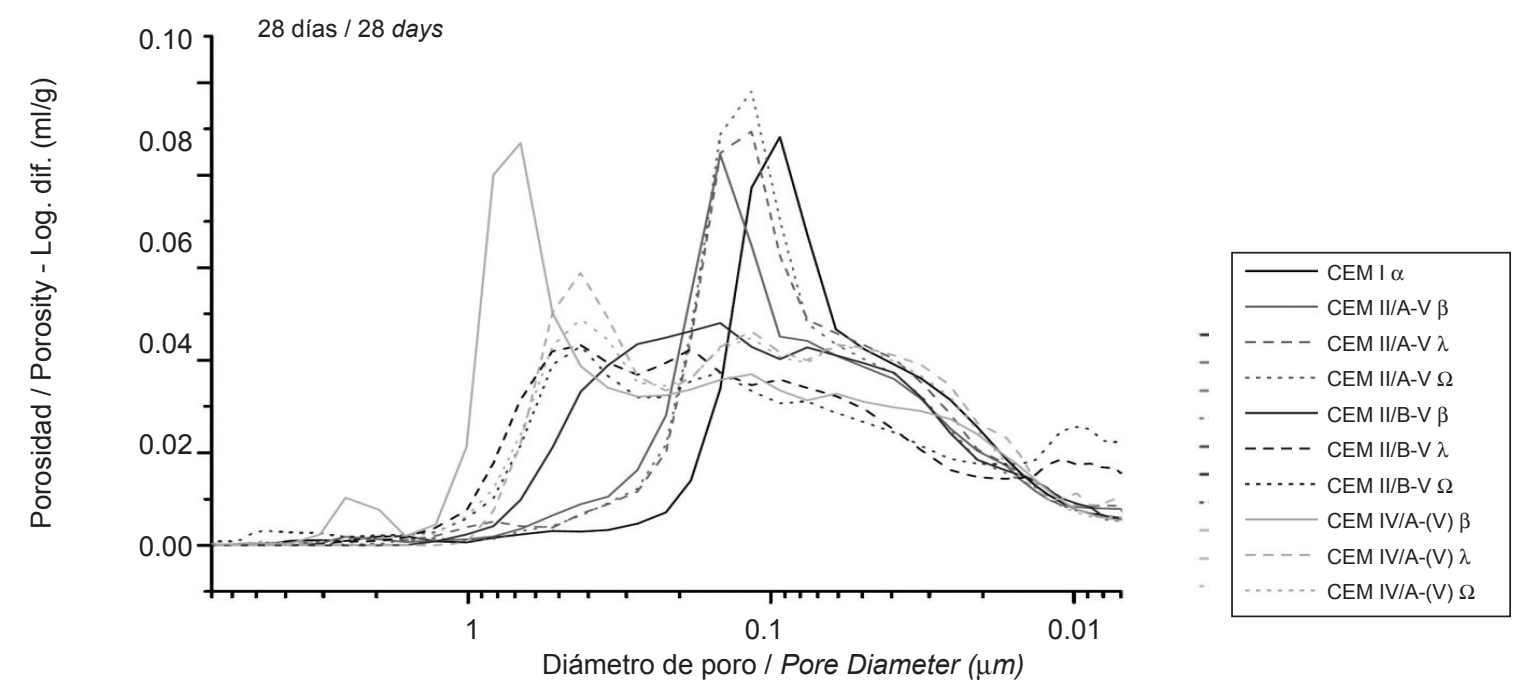

Figura 5. Porosidad a 28 días de los morteros de adición simulando cementos CEM II/A-V*, CEM II/B-V* y CEM IV/A (V*) con cenizas volantes silíceas $(\mathrm{V}), \beta$, y cenizas de fondo $(\mathrm{C}), \Omega$, y morteros con una mezcla $1: 1$ de ambas cenizas, l, en comparación con el cemento sin adiciones CEM I 42,5 N ( $\alpha$ ), determinada mediante porosimetría de intrusión de mercurio (MIP).

Figure 5. Open porosity at 28 days of mortars made of blended cements prepared in laboratory CEM II/A-V*, CEM II/B-V* and CEM $I V / A\left(V^{*}\right)$ using fly ash $(V), \beta$, bottom ash $(C), \Omega$, and mortars with a 1:1 (fly ash : bottom ash) mix, $(\alpha)$, in comparison to the cement without any addition CEM I $42.5 \mathrm{~N}$, determined by mercury intrusion porosimetry (MIP).
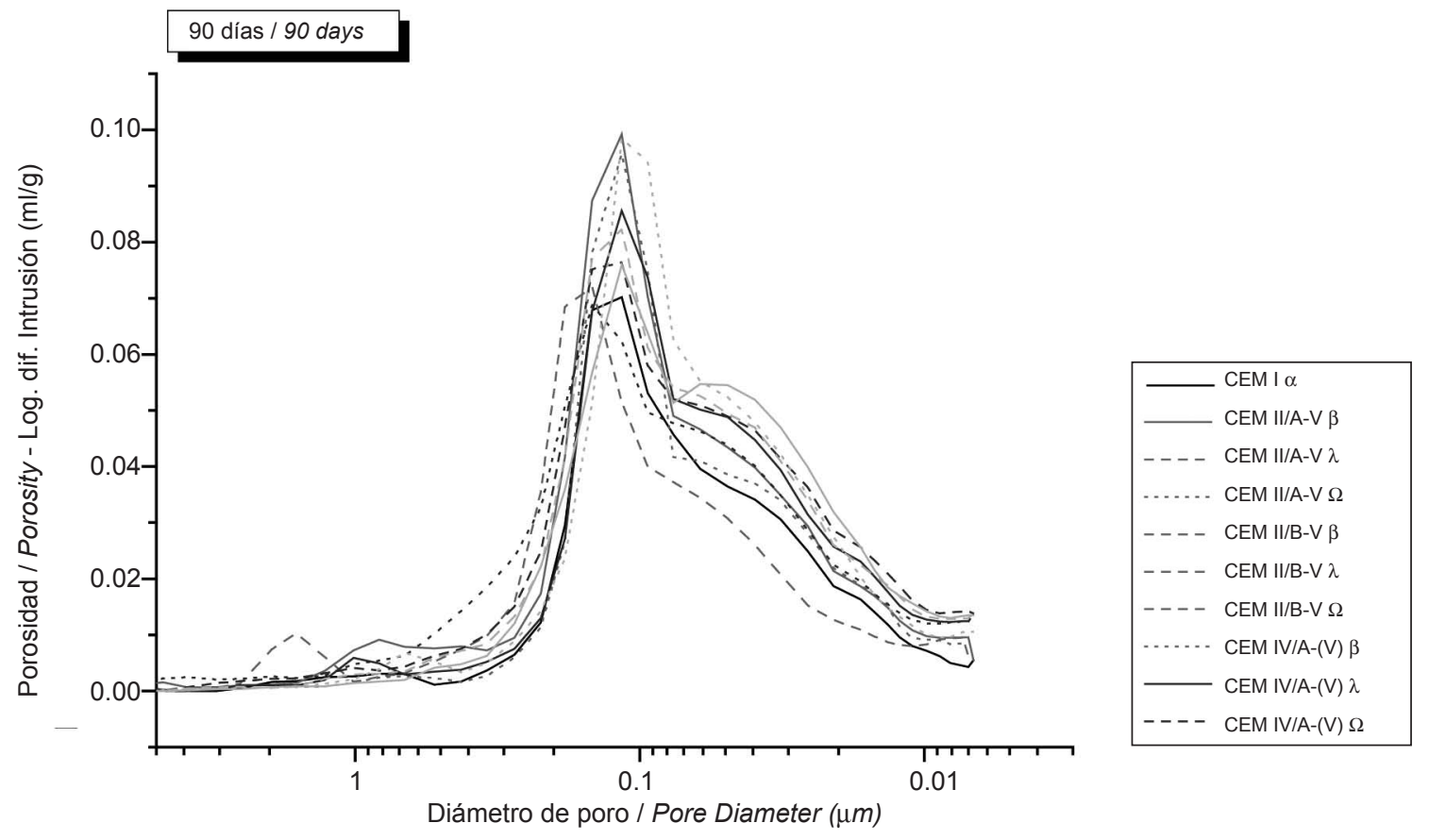

Figura 6. Porosidad a 90 días de los morteros de adición simulando cementos CEM II/A-V*, CEM II/B-V* y CEM IV/A (V*) con cenizas volantes silíceas $(V), \beta$, y cenizas de fondo $(C), \Omega$, y morteros con una mezcla 1:1 de ambas cenizas, $\lambda$, en comparación con el cemento sin adiciones CEM I 42,5 N ( $\alpha$ ), determinada mediante porosimetría de intrusión de mercurio (MIP). Figure 6. Open porosity at 90 days of mortars made of blended cements prepared in laboratory CEM II/A-V*, CEM II/B-V* and CEM IV/A(V*) using fly ash (V), $\beta$, bottom ash (C), $\Omega$, and mortars with a 1:1 (fly ash : bottom) ash mix, $\lambda$, in comparison to the cement without any addition CEM I $42.5 \mathrm{~N}(\alpha)$, determined by mercury intrusion porosimetry (MIP). 


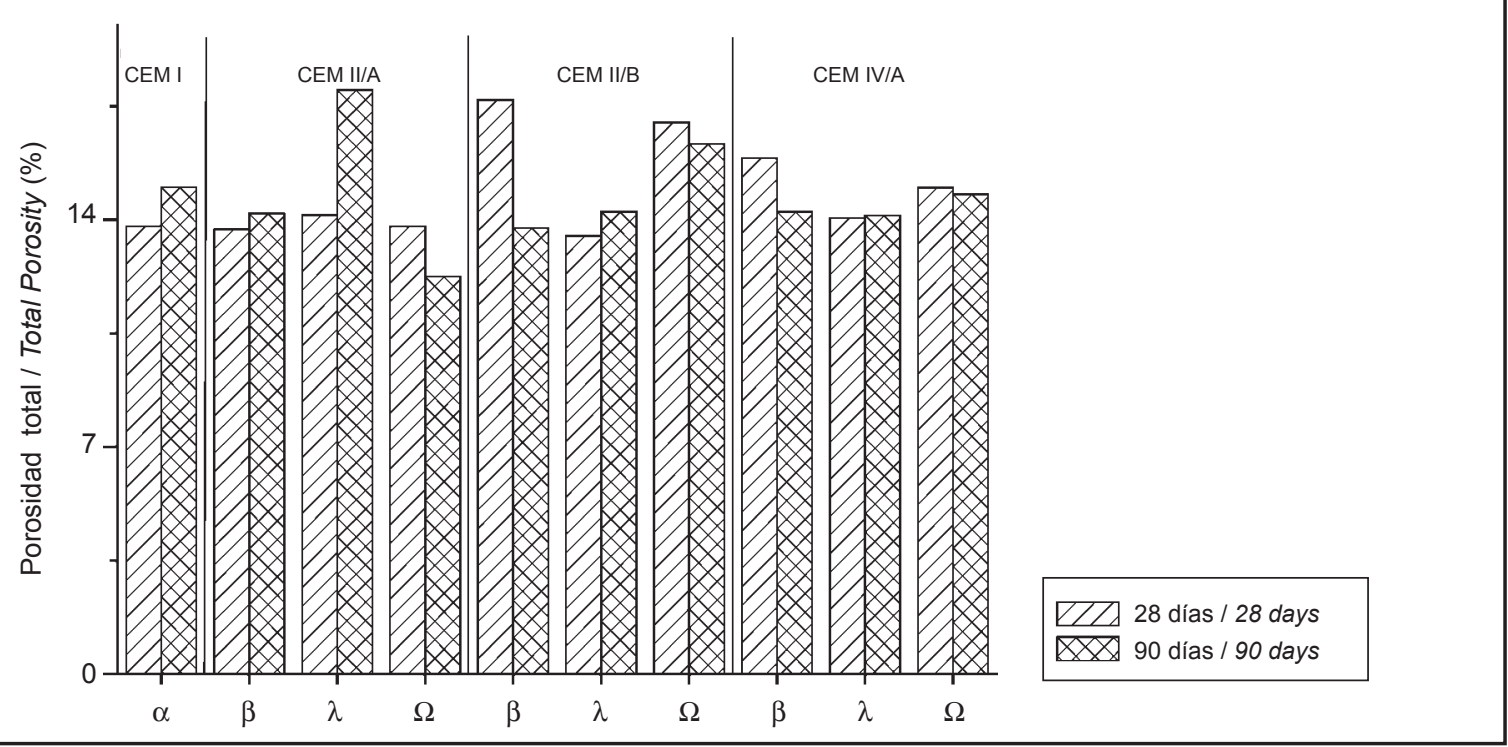

Figura 7. Porosidad total a 28 y 90 días de los morteros de adición simulando cementos CEM II/A-V*, CEM II/B-V* y CEM IV/A (V*) con cenizas volantes silíceas (V), $\beta$, y cenizas de fondo $(C), \Omega$, y morteros con una mezcla 1:1 de ambas cenizas, $\lambda$, en comparación con el cemento sin adiciones CEM I 42,5 N ( $\alpha$ ), determinada mediante porosimetría de intrusión de mercurio (MIP).

Figure 7. Total porosity at 28 days and 90 days of mortars made of blended cements prepared in laboratory CEM II/A-V*,CEM II/B-V* and CEM IV/A( $\left.V^{*}\right)$ using fly ash (V), $\beta$, bottom ash (C), $\Omega$, and mortars with a 1:1 (fly ash : bottom) ash mix, $\lambda$, in comparison to the cement without any addition CEM I $42.5 \mathrm{~N}(\alpha)$, determined by mercury intrusion porosimetry (MIP).

\section{DISCUSIÓN}

\subsection{Resistencias mecánicas a flexión y a compresión}

Una forma indirecta de evaluar la capacidad puzolánica de una ceniza es a través del aumento de la resistencia de los morteros elaborados con ésta a lo largo del tiempo en comparación con un mortero de referencia sin ceniza. Ambas cenizas, volante y de fondo, son puzolánicas de acuerdo con la norma europea EN 1965:2011, por un lado, pero también se confirma con los resultados de resistencia mecánica obtenidos. En un estudio similar (7) pero con una ceniza de fondo de un carbón de bajo poder calorífico $(4.500 \mathrm{kcal} / \mathrm{kg})$ y con un contenido en cenizas (42\%) muy superior al del carbón surafricano $(15 \%)$, se obtienen resultados similares de composición química en cuanto a los óxidos mayoritarios: $\mathrm{SiO}_{2}(56 \%), \mathrm{Al}_{2} \mathrm{O}_{3}(27 \%)$ y $\mathrm{Fe}_{2} \mathrm{O}_{3}(6 \%)$; sin embargo, se encuentran diferencias importantes en cuanto al contenido de $\mathrm{CaO}(0,8 \%)$ y pérdida por calcinación $(4,6 \%)$, todos ellos medidos por FRX. La ceniza de Carboneras tiene un contenido de $\mathrm{CaO}$ superior tanto en la ceniza volante $(5,0 \%)$ como en la de fondo $(5,9 \%)$, este hecho puede favorecer la reacción puzolánica de la sílice de la ceniza con el $\mathrm{Ca}(\mathrm{OH})_{2}$ liberado en la hidratación del cemento Portland común. Por otro lado, la reducida pérdida por calcinación de la ceniza volante de Carboneras $(3,6 \%)$ como en la de fondo

\section{DISCUSSION}

\subsection{Compressive and flexure strengths}

An indirect way to evaluate the pozzolanic activity of any coal ash is by means of the mortar strength increase elaborated with this ash along the time in comparison to a standard mortar without addition. Both ashes, fly ash and bottom ash, are pozzolanic materials according to the European standard EN 196-5:2011, but also, the pozzolanic activity can be evidenced by the mechanical strength results obtained. In a similar study (7), but using a low heat coal $(4500 \mathrm{kcal} / \mathrm{kg})$ and with an ash content of $42 \%$, much higher than South African coal used in the present study (15\%), similar results were obtained with regard to the chemical composition of the main oxides: $\mathrm{SiO}_{2}$ (56\%), $\mathrm{Al}_{2} \mathrm{O}_{3}$ (27\%) y $\mathrm{Fe}_{2} \mathrm{O}_{3}$ (6\%). However, significant differences were found with regard to $\mathrm{CaO}$ content $(0.8 \%)$ and loss on ignition (4.6\%), determined by XRF. Carboneras fly ash and bottom ash have a higher CaO content of $5.0 \%$ and $5.9 \%$, respectively. This result may improve the pozzolanic reaction of the silica present in the ashes with the $\mathrm{Ca}(\mathrm{OH})_{2}$ released from the hydration reaction of the Portland cement. On the other hand, the low loss on ignition of the Carboneras fly ash (3.6\%) as well as bottom ash (1.8\%) will have a positive effect on the durability of the binders made of them in cold environments (good frost-thaw resistance). As expected, 
$(1,8 \%)$, tendrá implicaciones favorables en la durabilidad de los materiales cementantes fabricados con ellas en ambientes fríos (buena resistencia al hielo-deshielo). Asimismo, como era de esperar, al aumentar la superficie específica de las cenizas de fondo mediante molienda, aumenta la reactividad puzolánica, además, también se puede atribuir a un cierto efecto de relleno $(7,16)$ y de igual manera se evidencia que a cortas edades la reacción puzolánica no ha tenido lugar en toda su extensión y, por tanto, existe una clara diferencia con el mortero de referencia $\alpha$ que se reduce con el paso del tiempo.

También era de esperar que la resistencia tanto a flexión como a compresión a 1 día del cemento de referencia sin adiciones CEM I 42,5 N $(\alpha)$ fuera ligeramente superior que la de los cementos CEM II/A-V*, CEM II/B-V* y CEM IV/A ( $\left.\mathrm{V}^{*}\right)$ independientemente de la mezcla de cenizas (volantes y de cenicero — de la $\beta$ a la $\Omega$ según la Tabla 2-). Esto es normal debido a que las adiciones puzolánicas como las cenizas volantes y de cenicero necesitan que los silicatos cálcicos del cemento reaccionen primeramente para formar $\mathrm{Ca}(\mathrm{OH})_{2}$ ya este es el promotor de la reacción puzolánica al reaccionar con la sílice reactiva de dichas cenizas. Sin embargo, estas diferencias no son significativas entre el cemento de referencia CEM I 42,5 N $(\alpha)$ sin adiciones y el cemento CEM II/A-V*, incluso a partir del primer día, independientemente del tipo de ceniza utilizado ( $\beta$ : $100 \%$ de cenizas volantes y $\Omega$ : $100 \%$ de cenizas de fondo); lo que demuestra inicialmente que la sustitución parcial o total de las cenizas volantes por las de cenicero en cementos de adición con un $10 \%$ de cenizas no tiene ningún efecto negativo en la resistencia a compresión ni en la resistencia a flexión. En general, se observa que a partir de 7 días la diferencia en las resistencias a compresión se ve más atenuada. Y que, a partir de los 14 días no hay diferencias significativas entre el cemento de referencia CEM I 42,5 $\mathrm{N}(\alpha)$ sin adiciones y el cemento CEM II/A-V*, independientemente del tipo de ceniza utilizado ( $\beta$ : $100 \%$ de cenizas volantes, $\Omega$ : $100 \%$ de cenizas de fondo) o mezclas de ambas.

Mullick et al. (17) han encontrado que con un $20 \%$ de cenizas de fondo molidas (>92\% inferior a $45 \mu \mathrm{m}$ ) y $80 \%$ de cemento Portland con un $0,338 \%$ de plastificante, la resistencia a compresión (14) es significativamente inferior a la de la mezcla que tiene cenizas volantes en vez de cenizas de fondo; por contra, esta tendencia se invierte a los 28 días dando valores de $29,0 \mathrm{MPa}$ y 41,1 MPa las mezclas 20:80 de cenizas volantes y de fondo, respectivamente; sin embargo, ambos valores siguen siendo inferiores a los del mortero de referencia sin cenizas (58,0 MPa). El orden de magnitud es similar al obtenido por los cementos CEM II/B-V* del presente trabajo a 28 días (CEM I 42,5 N ( $\alpha$ ): $55 \mathrm{MPa} ; \beta$ con el $100 \%$ de cenizas volantes: $60 \mathrm{MPa}$ y $\Omega$ con el $100 \%$ de the higher ash specific surface obtained by grinding, the higher pozzolanic activity. This might be also attributed to a filling effect $(7,16)$. At early ages, it is also evidenced the pozzolanic reaction was not produced completely, and then, it exist a clear difference with the mortar without addition $(\alpha)$, which is reduced with time.

As expected, compressive strength and flexure strength at one day of CEM I $42.5 \mathrm{~N}(\alpha)$, without additions, was slightly higher than that of CEM II/A-V*, CEM II/B-V* and CEM IV/A $\left(V^{*}\right)$ cements. The fly ashes-bottom ashes mixes (from $\beta$ to $\Omega$ according to Table 2) did not affect the results. This fact is explained due to the necessity of $\mathrm{Ca}(\mathrm{OH})_{2}$ by the pozzolanic additions (fly ashes and bottom ashes), which is formed when calcium silicates $\left(C_{3} S\right.$ and $\left.\mathrm{C}_{2} \mathrm{~S}\right)$ react with water. Calcium hydroxide $\left(\mathrm{Ca}(\mathrm{OH})_{2}\right)$ reacts with the reactive silica present in those ashes. However, these differences are not significant with regard to the reference cement CEM I $42.5 \mathrm{~N}(\alpha)$ without additions, and cement CEM II/A-V*, even after 24 hours, independently of the type of ashes used $(\beta: 100 \%$ fly ash and $\Omega: 100 \%$ bottom ash). This shows initially that partial or full replacement of fly ash by bottom ash in cement with less of $10 \%$ of ashes does not imply any negative effects in compressive or flexure strength. In general, it is observed that after 7 days, the difference in compressive strength is becoming shorter. And also, after 14 days, no significant differences between CEM I $42.5 N(\alpha)$ and CEM II/A-V* were found for all the fly ash-bottom ash mixes studied ( $\beta$ : $100 \%$ fly ash, $\Omega$ : $100 \%$ bottom ash; and mixes of them).

Mullick et al (17) found that using $20 \%$ ground bottom ash (>92\% was lower than $45 \mu \mathrm{m}$ ) and $80 \%$ of Portland cement with $0.338 \%$ of superplasticiser, the compressive strength (14) is significantly lower to that of the mix made of fly ash instead of bottom ash. On the contrary, this trend is reversed at 28 days; 20:80 (fly ash : bottom ash) mixes presented results of 29.0 MPa and 41.1 $\mathrm{MPa}$, respectively. However, both values are still being lower than the value of the mortar without addition (58.0 $M P a)$. The order of magnitude is similar to values obtained at 28 days in cements CEM II/B-V* of this paper (CEM I $42.5 \mathrm{~N}(\alpha)$ : $55 \mathrm{MPa} ; \beta=100 \%$ fly ash: $60 \mathrm{MPa}$ and $\Omega=100 \%$ bottom ash: $53 \mathrm{MPa})$. A difference with the results of the Mullick et al (17) paper, is that fly ash 
cenizas de fondo: $53 \mathrm{MPa}$ ). Pero al contrario de lo que sucede en el trabajo citado, ambas cenizas se comportan de una forma similar, aunque aparentemente la resistencia es ligeramente superior en el caso de la ceniza de fondo.

En los cementos CEM II/B-V* y CEM IV/A ( $\left.V^{*}\right)$, con un $25 \%$ y un $35 \%$ de cenizas, respectivamente, se obtienen en general unas resistencias a compresión menores a edades desde 7 a 90 días. Por el contrario, aunque esta tendencia sí se cumple en la resistencia a flexión a edades desde los 3 a los 28 días, se da de forma más atenuada y se rompe para la edad de 90 días.

En el análisis del efecto de la sustitución parcial o total de las cenizas volantes por las de cenicero en los cementos de adición con relación a la resistencia a flexión se puede decir que, teniendo en cuenta que la repetibilidad y la reproducibilidad del ensayo de resistencia a flexión expresadas como coeficiente de variación es del 4,0\% y 7,0\%, respectivamente, de acuerdo con los valores obtenidos en los últimos 15 años en el programa de ensayos interlaboratorios de cementos del IECA (18), no se aprecian diferencias significativas entre ambas cenizas. Por otro lado, en el análisis del efecto de la sustitución parcial o total de las cenizas volantes por las de cenicero en cementos de adición en cuanto a la resistencia a compresión en los cementos CEM II/A-V* y CEM IV/A ( $\left.V^{*}\right)$, con el menor y el mayor contenido de cenizas a cualquier edad, se puede decir que, considerando que la precisión del método de ensayo medida a través de la repetibilidad y reproducibilidad expresadas como coeficiente de variación de acuerdo con la norma europea UNE-EN 196-1:2005 (14) es del 2,0\% y 4,0\%, respectivamente, no se aprecian diferencias significativas entre ambas cenizas. Sin embargo, en el CEM II/B-V*, si bien no se aprecian diferencias significativas a 1 y 3 días, sí se observan éstas a partir del séptimo día hasta el nonagésimo. Es decir, se observan unos valores superiores de resistencia a compresión en las mezclas de cenizas con un mayor contenido de cenizas volantes $(\beta)$ en detrimento de las de cenicero $(\Omega)$. Este hecho puntual estaría asociado al diferente desarrollo microestructural de los morteros estudiados.

Asimismo, comparando los resultados de resistencia a compresión de las mezclas de los cementos CEM II/A-V*, CEM II/B-V* y CEM IV/A ( $\left.V^{*}\right)$ independientemente de la mezcla de cenizas (volantes y de cenicero - de la $\beta$ a la $\Omega$ según la Tabla $2-$ ) se evidencia que cumplen con los requisitos de resistencia a compresión a 2 y 28 días de la norma europea EN 197-1:2011. Por tanto, en este sentido, podrían incluirse para su uso como componente principal de los cementos Portland comunes en dicha norma. and bottom ash performed in a quite similar way in the present research study. Only a slight higher strength was found in some bottom ash mortars.

Compressive strength of CEM II/B-V* and CEM IV/A $\left(V^{*}\right)$, with $25 \%$ and $35 \%$ of ashes, respectively, was becoming lower from 7 to 90 days. On the contrary, this trend was only observed from 3 to 28 days with regard to flexure strength, but it was broken at 90 days.

Summing up, taking into account the repeatability and reproducibility of the test method after the analyses performed with regard to the flexure strength of mortars with fly ash, bottom ash or mixtures of them, it can be said that no differences can be found between both ashes (bottom and fly ashes). Repeatability and reproducibility of flexure testing method are $4.0 \%$ and $7.0 \%$ expressed as variation coefficient, respectively, according to the values obtained during the last 15 years in the interlaboratory test program developed by IECA (18). On the other hand, with regard to the compressive strength of the CEM II/A-V* and CEM IV/A $\left(V^{*}\right)$, it can be concluded that, taking into account the repeatability and reproducibility of the test method, no differences can be found between both ashes (bottom and fly ashes). The trueness values of the compressive strength testing method, repeatability and reproducibility expressed as variation coefficient are $2.0 \%$ and $4.0 \%$, respectively, according to the Spanish standard UNE-EN 196-1: 2005 (14), equivalent to the European standard EN 196-1. However, CEM II/B-V* presented no significant differences at 1 and 3 days, but from 7 to 90 days important differences were found. That is, the higher fly ash ( $\beta$ ) content, the higher compressive strength in mortars with mixes made of both ashes (fly ash and bottom ash). This isolated observation may be connected to the peculiar mortar microstructural development.

Also, comparing compressive strength results of ash mixes employed to prepare CEM II/A-V*, CEM II/B-V* and CEM IV/A $\left(V^{*}\right)$, independently of the ash mix (fly ash -from $\beta$ to $\Omega$ according to Table 2-), it can be realised that comply with the requirements of 2 days and 28 days according to EN 197-1:2011. Therefore, these materials could be included as a main constituent of common cements in mentioned standard. 


\subsection{Porosidad de los morteros}

La porosidad disminuye con la edad de curado de forma general (Figura 4), siendo esta disminución más significativa en el cemento de referencia sin adiciones CEM I $42,5 \mathrm{~N}(\alpha)$. De forma general, se observa que la porosidad a 28 días es menor en las mezclas con sustitución del $10 \%$ con cenizas volantes, de cenicero o mezclas de ambas; mientras que para mezclas con una sustitución del $25 \%$ y del $35 \%$ de adición por cemento la porosidad a los 28 días está en valores del mismo orden que el cemento de referencia CEM I 42,5 N ( $\alpha$ ), si bien a los 90 días la porosidad total es algo mayor que en el cemento de referencia; esto es más acusado cuanto mayor es la sustitución de cemento, es decir, cementos CEM IV/A ( $\left.V^{*}\right)$. También se observa que en los cementos con mayor sustitución de cemento por cenizas CEM IV/A ( $\left.\mathrm{V}^{*}\right)$ la disminución de la porosidad entre los 28 días y los 90 días es menor que para las menores proporciones de sustitución, lo que indica una optimización de la densificación de los productos de hidratación para las mezclas con sustituciones del $10 \%$ de adición (CEM II/A- $\mathrm{V} *$ ), independientemente del tipo de ceniza (volante o cenicero) que se utilice como sustitución. Incluso es de destacar que para sustituciones del $10 \%$ y del $25 \%$, cementos CEM II/A-V* y CEM II/B-V*, respectivamente, la porosidad a 90 días es menor para la sustitución con ceniza de cenicero sólo, con relación a la ceniza volante o la mezcla de ambas.

Si analizamos distintos intervalos de tamaños de poros se observa una mayor presencia de poros capilares medianos (0,01-0,05 $\mu \mathrm{m})$, en los morteros con mayor sustitución de cemento (CEM IV/A $\left(V^{*}\right)$ ). Si bien estas diferencias se atenúan a 90 días en los que se queda en valores similares a los cementos CEM II/B-V*, lo que se asocia con la evolución más lenta de los productos de hidratación de las partículas de cenizas en los materiales con mayor sustitución de cemento por cenizas volantes o cenizas de cenicero.

Por su parte, los poros con tamaños situados entre $0,05-$ $0,1 \mu \mathrm{m}$ se mantienen prácticamente estables después de los 28 días en todas las mezclas estudiadas, debido a que los productos de hidratación formados son de menor tamaño $(0,01-0,05 \mu \mathrm{m})$ o de mayor tamaño $(0,1-0,5 \mu \mathrm{m})$. También, es significativa la práctica desaparición de los poros con tamaños comprendidos entre $0,1-0,5 \mu \mathrm{m}$ a los 90 días, para todas las muestras estudiadas, lo que se asocia con el relleno de estos huecos debido a la evolución de los productos de hidratación (19), observándose que con el cemento de referencia CEM I 42,5 N se produce la mayor disminución de estos poros (20).

Por otra parte, los poros de tamaño mayor ( $>1 \mu \mathrm{m}$ ) disminuyen en general en todas las mezclas, entre 28 días y 90 días, como consecuencia de la evolución de la hidratación.

\subsection{Mortar porosity}

Porosity decreases with curing time in general (Figure 4). This decrease is more evident with cement without additions CEM I $42.5 \mathrm{~N}(\alpha)$. In general, it is observed that porosity at 28 days is lower in mixes with a 10\% cement replacement with fly ash, bottom ash or a mix of both of them. While for a $25 \%$ or $35 \%$ cement replacement, porosity values at 28 days are similar to that of CEM I $42.5 \mathrm{~N}(\alpha)$; but at 90 days, total porosity is slightly higher than in CEM I $42.5 \mathrm{~N}$. This performance is stronger when the cement replacement increases, e.g. CEM IV/A ( $\left.V^{*}\right)$. It is also observed that cements having the highest cement replacement by ashes, CEM IV/A $\left(V^{*}\right)$, the porosity decreasing from 28 to 90 days is lower than in other cases with a lower cement replacement. This fact shows a densification due to the hydration products for mixes with $10 \%$ cement replacement (CEM II/A-V*), independently of the ash type (Bottom ash-Fly ash) used as replacement. It can even be highlighted that $10 \%$ and $25 \%$ replacement, CEM II/A-V* and CEM $I I / B-V^{*}$, respectively, porosity at 90 days is lower when only bottom ash are used as replacement, in comparison to fly ash replacement or even a Bottom ash-Fly ash mix replacement.

Analysing the pore size distribution, separated by intervals of size, a higher presence of medium capillary pores (0.01-0.05 $\mu \mathrm{m})$ in mortars with the highest cement replacement (CEM IV/A $\left.\left(V^{*}\right)\right)$ is observed. Such differences decrease at 90 days reaching porosity values similar to those of CEM II/B-V*. This is a clear consequence of the slower evolution of hydration products of ashes in mortars with a higher amount of fly ash or bottom ash.

With regard to pore sizes of 0.05-0.1 $\mu \mathrm{m}$, after 28 days remains almost constant for all the studied mortar mixes. This is due to the smaller size of hydration products formed (0.01-0.05 $\mu \mathrm{m})$ or higher size $(0.1-0.5 \mu \mathrm{m})$. It also can be mentioned the dramatically decrease of pores in the range of 0.1-0.5 $\mu \mathrm{m}$ at 90 days in all the mortar samples studied. This effect is explained by the filling effect as result of hydration products evolution (19). Such effect is more evident in mortars made of CEM I $42.5 \mathrm{~N}$ without any addition (20).

On the other hand, a general decrease of greater pores (>1 $\mu \mathrm{m}$ ) in all the mixes from 28 to 90 days was found as a result of the hydration products formation. 
Analizando la evolución de los poros capilares grandes en el rango de 0,5-1,0 $\mu \mathrm{m}$ se observa una disminución general de poros a los 90 días, asimismo se aprecia que hay más cantidad de estos poros en las mezclas con mayor cantidad de ceniza de cenicero con relación a la ceniza volante, produciéndose una disminución más significativa en estos casos a los 90 días.

De acuerdo con el análisis microestructural de las mezclas se observa, de forma general, que la evolución de la hidratación de las mezclas ensayadas hace que se produzca una disminución de los capilares medianos $(0,01-0,05 \mu \mathrm{m})$, los capilares grandes en el rango de 0,5 $-1,0 \mu \mathrm{m}$ y los poros grandes mayores de $1 \mu \mathrm{m}$ y que prácticamente desaparezcan los capilares grandes en el rango de 0,1-0,5 $\mu \mathrm{m}$. Específicamente se observa que la evolución de la hidratación hace que disminuyan los poros capilares medianos $(0,01-0,05 \mu \mathrm{m})$ especialmente en las mezclas con más sustitución de cemento por cenizas (volantes y/o de cenicero) y que prácticamente desaparecen los poros capilares grandes en el rango de 0,1-0,5 $\mu \mathrm{m}$ en todas las mezclas. Esto indica que la presencia de adiciones en mayor cantidad produce una estructura más cerrada en los poros capilares de tamaño mediano $(0,01-0,05 \mu \mathrm{m})$. Por su parte, en los poros capilares grandes en el rango de 0,5-1,0 $\mu \mathrm{m}$ se observa que los morteros con mayor sustitución de cenizas de cenicero con relación a las cenizas volantes, para un mismo porcentaje de sustitución total, tienen una porosidad algo mayor cuanto mayor es el porcentaje de sustitución de cenizas de cenicero (0\%-100\% y $50 \%-50 \%$, con respecto a $100 \%-0 \%$ ). En cuanto a la porosidad total, el cemento de referencia $(\alpha)$ presenta unos valores muy parecidos a los del cementos CEM II/A-V*- $\beta$ (sólo cenizas volantes) tanto a 28 como a 90 días y del mismo orden de magnitud que el CEM II/A$V^{*}$ - $\Omega$ (sólo cenizas de fondo); sin embargo, la mezcla de ambas cenizas $(\lambda)$ hace que la porosidad total a 90 días sea muy superior. Este efecto de las mezclas $\lambda$ no se repite ni en los CEM II/B-V* ni en los CEM IV/A ( $\left.V^{*}\right)$. En general, no se detecta ningún efecto determinante en la porosidad total de los morteros normalizados que se sitúa en torno al $14 \%$ con algunos picos puntuales que pueden llegar al $18 \%$ y con valores ligeramente superiores en todos los CEM IV/A ( $\left.\mathrm{V}^{*}\right)$ que contienen un $35 \%$ de cenizas.

\section{CONCLUSIONES}

De acuerdo con el análisis de las características mecánicas de resistencia a flexión y compresión, de las mezclas estudiadas se observa que el efecto de la sustitución parcial o total de las cenizas volantes por las cenizas de cenicero en los cemento de adición no incide produciendo diferencias significativas entre ambas cenizas,
Analysing big capillary porosity evolution among the 0.5 1.0um range, a general pore size decrease is observed at 90 days. Moreover, a greater amount of these pores is observed when a higher amount of bottom ash than fly ash is used. This effect is reduced at 90 days.

According to the microstructural analysis, hydration promotes a decrease of medium capillary pores (0.01$0.05 \mu \mathrm{m})$, big capillary pores $(0.5-1.0 \mu \mathrm{m})$ and big pores higher than $1 \mu \mathrm{m}$. Particularly, hydration reaction products fill in medium capillary pores (0.01-0.05 $\mu \mathrm{m})$, especially, in mixes with the higher amount of ashes (bottom ash, fly ash or a mix of them). Almost, big capillary pores disappear (0.1-0.5 $\mu \mathrm{m}$ ) for all the mixes. This fact shows that the presence of additions in a higher amount promotes a denser and closer microstructure in medium size capillary pores $(0.01-0.05 \mu \mathrm{m})$. On the other hand, in big capillary pores (0.5-1.0 $\mu \mathrm{m})$, mortars with more bottom ash than fly ash replacement have a slightly higher porosity. For an equal ash replacement, the porosity increases when the amount of bottom ash also increases (0\%-100\% and 50\%-50\% with regard to $10 \%-0 \%)$. With regard to the total porosity at 28 and 90 days, CEM I $42.5 \mathrm{~N}(\alpha)$ and CEM II/A-V* ( $\beta$ : $100 \%$ Fly ash) presented similar values. These values were of the same magnitude as CEM II/A-V* ( $\Omega$ : $100 \%$ Bottom ash). However, the 1:1 (fly ash : bottom ash) mix ( $\lambda$ ) promotes a higher total porosity at 90 days. This effect found in 1:1 Fly ash:Bottom ash mixes was not recorded in CEM $I I / B-V^{*}$ or CEM IV/A ( $\left.V^{*}\right)$. In general, it can be said that no significant effect was found in the total porosity of standardised mortars (about 14\%). Rarely, some peaks could reach values of $18 \%$. These values were slightly higher in CEM IV/A $\left(V^{*}\right)$ with $35 \%$ of ashes.

\section{CONCLUSIONS}

According to the mechanical characteristics studied (compressive and flexure strength) of the tested mortar mixes, it is observed that partial or full replacement of cement by fly ash compared to a similar replacement by bottom ash in blended cement does not produce relevant differences between both ashes. The slight occasional 
interpretándose alguna pequeña diferencia debido a la variabilidad propia del método de ensayo.

El análisis microestructural de las distintas mezclas indica que el mayor porcentaje de substitución de cemento por cenizas hace que disminuya la porosidad capilar pequeña $(0,01-0,05 \mu \mathrm{m})$ y que la mayor substitución de ceniza de cenicero por ceniza volante hace que la porosidad capilar alta $(0,5-1,0 \mu \mathrm{m})$ sea algo mayor que en las mezclas con el mismo porcentaje de sustitución con más ceniza volante.

Como conclusión general se puede decir que la utilización de las cenizas de fondo o cenicero mezcladas con cenizas volantes en los porcentajes estudiados del $0 \%$ al $100 \%$ no influye significativamente en el comportamiento mecánico de los morteros estudiados en los que el contenido máximo de adición era del 35\%, considerando que la repetibilidad y la reproducibilidad del ensayo de resistencia a flexión es del $4,0 \%$ y 7,0\%, expresadas como coeficiente de variación, y del $2,0 \%$ y $4,0 \%$, en cuanto al ensayo de resistencia a compresión, respectivamente, se puede concluir que no se aprecian diferencias tecnológicas significativas entre ambas cenizas. Este hecho hace que puedan incorporarse como componente principal en la próxima revisión de la norma europea de cementos comunes EN 197-1:2011.

\section{AGRADECIMIENTOS}

Los autores agradecen al Instituto Español del Cemento y sus Aplicaciones (IECA) la financiación de los ensayos a través del contrato de investigación firmado con el Instituto de Ciencias de la Construcción "Eduardo Torroja" (CSIC) relativo a la "caracterización físico-química durable de materiales en base cemento con incorporación de subproductos industriales" (contrato AU 23-10). differences found may be attributed to the variability of the experimental procedures or testing methods.

The microstructural analyses of the different mixes showed that the highest percentage of cement replacement by ashes produces a decrease of small capillary pores (0.01-0.05 $\mu \mathrm{m})$. Also, the highest amount of bottom ash with regard to fly ash promotes slightly higher big capillary porosity $(0.5-1.0 \mu \mathrm{m})$.

As general conclusion, it can be said that bottom ash mixed with fly ash in percentages from $0 \%$ to $100 \%$ do not affect significantly on the mechanical performance of the studied blended mortars. These mortars had a highest amount of additions of 35\%. Taking into account that repeatability and reproducibility of flexure strength testing are $4.0 \%$ and $7.0 \%$ expressed as variation coefficient, and $2.0 \%$ and $4.0 \%$, with regard to the compressive strength testing, respectively, it can be concluded that there are not technological differences between both ashes. This fact suggests that bottom ash could be included as a main constituent in the next revision of the European common cement standard EN 197-1:2011.

\section{ACKNOWLEDGMENT}

Funding for the trials conducted was provided by the Instituto Español del Cemento y sus Aplicaciones (IECA, Spanish institute for cement and its applications) under a research contract (agreement AU 23-10) concluded with the Eduardo Torroja Institute for Construction Science (a Spanish National Research Council body) in connection with "Durable physico-chemical characterization of cement-based materials with industrial by-products".

\section{BIBLIOGRAFÍA / BIBLIOGRAPHY}

(1) EN 197-1:2011. Cement - Part 1: Composition, specifications and conformity criteria for common cement.

(2) UNE-EN 197-1:2000 - Cemento. Parte 1: Composición, especificaciones y criterios de conformidad de los cementos comunes. 29 pp. + UNE-EN 197-1:2000 erratum - Cemento. Parte 1: Composición, especificaciones y criterios de conformidad de los cementos comunes. 7 pp.

(3) Anuarios del 2005 al 2010 de Oficemen (Agrupación de Fabricantes de Cemento de España). Madrid.

(4) Taylor, H. F. W.: Cement chemistry, p. 459, Thomas Telford, London, 1997. Edición 2a (1997).

(5) Menéndez, E.; de Frutos, J.: "Ion monitoring solutions in cements using electrical spectroscopy", Revista Mexicana de Física, vol. 55, no 1/2009, pp. 76-80.

(6) Menéndez, E; de Frutos, J.; Andrade, C.: "Internal deterioration of mortars in freeze-thawing: non-destructive evaluation by means of electrical impedance", Advanced Materials Research (2009), vol. 68, pp. 1-11.

(7) Cheriaf, M.; Rocha, J. C.; Pera, J.: "Pozzolanic properties of pulverized coal combustion bottom ash", Cem. Concr. Res. (1999), 29, pp. 1387-1391.

(8) Bai, Y.; Bashaer, P. A.: "Influence of furnace bottom ash on properties of concrete", Proceedings of the Institution of Civil Engineers. Structures and Buildings (2003), 156 (1), pp. 85-92.

(9) Pera, J.; Coutaz, L.; Ambroise, J.; Chababbet, M.: "Use of incinerator bottom ash in concrete", Cem. Concr. Res. (1997), 27 (1), pp. 1-5. 
(10) Churcill, V. E.; Amirkhanian, S. N.: "Coal ash utilization in asphalt concrete mixtures", Journal of Materials in Civil Engineering (1999), $11(4)$, pp. 295-297.

(11) EPA. "Study on Increasing the Usage of Recovered Mineral Components in Federally Funded Projects Involving Procurement of Cement or Concrete to Address the Safe, Accountable, Flexible, Efficient Transportation Equity Act: A Legacy for Users". United States Environmental Protection Agency in conjunction with the U.S. Department of Transportation and the U.S. Department of Energy. Report to Congress. June 3, 2008. United States Environmental Protection Agency Washington, D.C. 20460. EPA530-R-08-007, p. 227.

(12) ECOBA, 2005. European Coal Combustion Products Association e.V. Annual Report 2005,14 pp.

(13) ACAA, 2007. Coal Combustion Product (CCP) Production \& Use Survey Results. ACAA 15200 E. Girard Ave.; Ste. 3050. Aurora, CO 80014. 9/15/2008, p. 1.

(14) UNE-EN 196-1:2005 - Métodos de ensayo de cementos. Parte 1: Determinación de resistencias mecánicas.

(15) UNE-EN 1936:2077. Métodos de ensayo para piedra natural. Determinación de la densidad real y aparente y de la porosidad abierta y total.

(16) Berry, E. E.: "Strength development of some blended cement mortars", Cem. Concr. Res. (1980), 10, pp. 1-11.

(17) Mullick, A. K.; Justnes, H.; Fidjestøl, P.; Harsh, S.: Ternary Cement blends for improved sustainability, no 016, 7 pp. Cementing a sustainable future, XIII ICCC International congress on the chemistry of cement - Abstracts and Proceedings. Madrid, 3-8 July, 2011. Editors: Ángel Palomo, Aniceto Zaragoza, Juan Carlos López Agüí. Published by Instituto de Ciencias de la Construcción "Eduardo Torroja", CSIC. ISBN: Complete work - 978-84-7292-399-7 / Cd 978-84-7292-400-0. @Autors (2011)

(18) IECA, 2011. Programa de ensayos interlaboratorios de cementos. Primer semestre del año 2011. EI-9811: CEM II/A-V 42,5 R. EI-9911: CEM II/A-P 42,5 R. REF.: 3CTC015/11-13.

(19) Chindaprasirt, P.; Jaturapitakkul, Ch.; Sinsiri, Th.: "Effect of fly ash fineness on microstructure of blended cement paste", Constr. Build. Mater., 21 (2007), pp. 1534-1541.

(20) González-Fonteboa, B.; Carro-López, D.; Gutiérrez, S.; Sánchez, M.: "Characterization of fly and bottom ash from a fluidized-bed power plant as an addition to cement", n० 678, 7 pp. Cementing a sustainable future, XIII ICCC International congress on the chemistry of cement - Abstracts and Proceedings. Madrid, 3-8 July, 2011. Editors: Ángel Palomo, Aniceto Zaragoza, Juan Carlos López Agüí. Published by Instituto de Ciencias de la Construcción "Eduardo Torroja", CSIC. ISBN: Complete work - 978-84-7292-399-7 / Cd 978-84-7292-400-0. (C)Autors (2011). 